on the part of the trypaflavin to move into the direction of the lymph vessels and also why there is not such a thing in the case of fluorescein. Not much has been written about the lymph vessels of the conjunctiva. There is just one paper.by Knüssel and Vonwiller, who succeeded in making the lymph vessels visible by staining with kresyviolet. This stain is also charged negatively in the blood. Furthermore Cuénod and Nataf (Bull. Soc. d' Ophtal. de France, 1934) show a picture which is also from Knüssel and Vonwiller in which some lymph vessels can be seen which are similar to those mentioned above. The method described in this paper is much more sensitive than any used previously.

\title{
THE CLINICAL VALUE OF TRYPTOPHANE- REACTION
}

BY

\section{J. A. van Heuven}

UTRECHT

A NUMBER of papers on the importance of the tryptophane-reaction in the diagnosis of meningitis tuberculosa has lately been published in various medical journals. A list of these papers was drawn up at the close of an article in the Zeitschr. f. Kinderheilk., Bd. LVI, Hft. 6, 1934, by H. M. Schumacher, who concluded that in every case of meningitis tuberculosa the reaction is positive, whereas it is invariably negative in any other case in which the liquor is not very cloudy or bloody or purulent.

In view of the close affinity between aqueous and liquor cerebri it would seem interesting to trace the course of this reaction on the aqueous in cases of ophthalmic tuberculosis, especially as a reaction quickly showing whether a process is of a tuberculous nature or not, would surely be useful for clinical purposes.

The tryptophane reaction is a simple one and does not present any serious practical difficulties; but ophthalmologists are a ways somewhat hampered by the exiguous quantity of the material available. Consequently in the first place it was necessary to ascertain whether the reaction could still be performed with a quantity of liquid of not more than 0.1 c.c. This limit has been set, as puncture of the anterior chamber always yields this minimum quantity. In the second place the degree of sensitiveness of the reaction must be made sure of, and therefore various solutions of pure tryptophane 
were made and on these the reaction was performed. A quantity of $10 / 1000$ m.g. appeared to be demonstrable in 0.1 c.c. of liquid.

The reaction is performed as follows: 1.5 c c. concentrated hydrochloric acid. are added to a quantity from 0.1 to 0.2 c.c. aqueous; followed by one drop of 2 per cent. solution of formalin; the mixture is shaken once.

Next the solution should be allowed to stand for five minutes, $\stackrel{\mathbb{D}}{\triangle}$ after which a thin layer of 0.2 c.c. of a 0.6 per cent. solution of $\&$ nitrate of sodium should be carefully added. After 2 or 3 minutes a violet ring will appear at the point of contact of the two liquids, at least when the reaction is positive. The solution of formalin should be prepared fresh for each day.

If the aqueous is normal the reaction is always negative both for rabbits and men.

I next inoculated the anterior chamber of rabbits with tubercle bacilli, performing also reactions with other species of bacilli for control purposes. A detailed description of these experiments appears superfluous, as in all cases of reactive inflammation the reaction proved positive. Therefore it seems that this reaction cannot be considered specific to tuberculosis, as the result of every reactive inflammation effected by me turned out positive.

The rabbits were subjected to prolonged clinical observation and the reaction was invariably found to be positive when the slit-lam 18 no longer revealed any symptoms of inflammation. Consequentlo it was out of the question to explain the positive reaction as being caused by the presence of additional blood or leucocytes. Neither could there exist any direct relation with the albumen percentage in the anterior chamber, as was proved by the cases in which the Tyndall-phenomenon in the aqueous was already entirely negative, whereas the reaction always turned out positive.

In explanation of the fact of the tryptophane-reaction being positive the following possibilities suggest themselves: Tryptophane could arise through the decomposition of containing tryptophane albumen, normally existing in the eye. Secondly the tryptophane might have been introduced by the blood. This could only be possible if the permeability of the capillaries should have hecome temporarily increased. The following experiments were performed in order to determine the necessary data :

In the first place the demonstrability of tryptophane in the bloodserum of rabbits was successfully ascertained : the albumen-fraction invariably contained tryptophane.

After puncture of the anterior chamber of a rabbit's eye normal aqueous, as has already been stated, always produces a negative reaction. After a second puncture one hour later, the reaction of this aqueous which contains a very considerable albumen-fraction is positive. It is well known that the albumen in the "second aqueous" 
is derived from the blood. By the first puncture the capillaries become permeable to albumen, as has been shown by Wessely, who, before the first puncture, injected adrenalin under the conjunctiva, with the result that the albumen-percentage did not show any increase. I have repeated this experiment but the tryptophanereaction proved positive notwithstanding the negative albumenreaction. From this may be concluded, in the first place: that the positive tryptophane-reaction is dependent on the albumenpercentage. Neri (in Rass. stud. psych., Vol. XXIII, p. 1139, 1934) stated the opposite. In the second place the tryptophane reaction might be assumed to be a particularly sensitive test of the permeability of the capillaries, even more than Kikai's staining reaction (Arch. f. Augenheilk., Vol. CIII). This is in complete concurrence with the fact that the tryptophane-reaction still appeared positive when the slit-lamp revealed the optical emptiness of the anterior chamber. It is a well-known fact that the permeability of the capillaries is chiefly determined by two factors: by the size of the particles and by their charge. These experiments therefore show that the size of the particles must be equal to or smaller than that of eosin and similarly charged. Theoretically there is an essential difference between these experiments and those in which extraneous substances as e.g., stains, are injected into the blood in order to test the permeability of the vessel. In the present instance a substance is used which normally exists in the circulation, so that objections which might otherwise be raised are removed beforehand.

Availing myself of the peculiar qualities of the tryptophanereaction in this respect $I$ have renewed the experiments related previously, concerning artificially generated oedema of the conjunctiva. During preliminary experiments the conjunctivae of rabbits, after being extirpated, were pounded in a mortar and a small quantity of distilled water was subsequently added. Allowing the liquid to stand for some time I performed the tryptophane-reaction on it. This reaction was always negative. When oedema was first artificially generated and next the conjunctiva subjected to the same treatment the reaction appeared to turn out positive. Naturally great care was taken to keep the extirpated conjunctiva free from blood-vessels, which owing to the anatomical relations of the conjunctiva did not offer peculiar difficulties. Thus the fact that artificially generated oedema increases the permeability of the vessels of the conjunctiva could be demonstrated.

Summarizing we may conclude that the tryptophane-reaction has been proved to be not a specific test of tuberculosis, but a very sensitive indication of the increased permeability of the capillaries. Moreover an advantage of the reaction is that it allows us to demonstrate increased permeability by means of a substance normally existing in the circulation. 